\title{
Trapping and Injecting Single Domain Walls in Magnetic Wire by Local Fields
}

\author{
Manuel Vázquez, * G. A. Basheed, Germán Infante, and Rafael P. Del Real \\ Materials Science Institute of Madrid, CSIC. 28049 Madrid. Spain
}

(Received 1 September 2011; published 18 January 2012)

\begin{abstract}
A single domain wall (DW) moves at linearly increasing velocity under an increasing homogeneous drive magnetic field. Present experiments show that the DW is braked and finally trapped at a given position when an additional antiparallel local magnetic field is applied. That position and its velocity are further controlled by suitable tuning of the local field. In turn, the parallel local field of small amplitude does not significantly affect the effective wall speed at long distance, although it generates tail-to-tail and head-to-head pairs of walls moving along opposite directions when that field is strong enough.
\end{abstract}

DOI: 10.1103/PhysRevLett.108.037201

PACS numbers: $75.60 . \mathrm{Ch}, 75.70 . \mathrm{Kw}$

Modern high-density ultrafast data storage and logic devices [1] involve field or current driven domain wall motion in magnetic wires [2,3]. While in racetrack memory devices, an entire encoded domain wall (DW) pattern moves coherently along a nanostrip [4], in logic devices single domain walls propagate through a magnetic nanostrip network containing various gates and junctions [5]. Consequently, a very precise control of DW motion is of crucial importance for the development of such advanced devices.

Nanostrip lithography wires are characterized by transverse and vortex DWs injected under suitable conditions $[6,7]$. DW motion is artificially pinned by local notches $[8,9]$ or stray fields [10] as has been directly observed [11]. Their velocity increases linearly with an applied field until reaching a breakdown where walls reduce speed and change shape [1,12]. Particularly, experimental [13] and simulation [14,15] studies have shown that transverse fields modify the DW velocity.

Nevertheless, no clue is achieved about the nucleation or control in position and velocity of a DW without irreversibly modifying the nanowire geometry (i.e., notches) or applying sufficiently controlled local fields (i.e., local stray fields or nonlocal transverse fields). Here we propose a new, simple, and more flexible way of injecting and trapping single domain walls, locate them in a given desired position or move them with high speed in the linear behavior with an applied field (i.e., no breakdown).

In order to demonstrate this technology we are considering the case of magnetic cylindrical wires, a few microns in diameter and up to kilometers in length. Amorphous glass-coated microwires, produced by the quenching and drawing technique, have been used in the present experiments, with $\mathrm{Fe}_{77.5} \mathrm{Si}_{7.5} \mathrm{~B}_{15}$ nominal composition, $11 \mathrm{~cm}$ in length, and an $11 \mu \mathrm{m}$ metallic core diameter covered by a Pyrex-glass coating to result in a total diameter of $40 \mu \mathrm{m}$. The alloy composition (saturation magnetic polarization, $\mu_{0} M_{s}=1.56 \mathrm{~T}$ ) was chosen so that the wire exhibits high $\left(3 \times 10^{-5}\right)$ magnetostriction and consequently strong longitudinal uniaxial magnetic anisotropy that results in a magnetic bistable character. As investigated in previous studies [16,17], a single-domain structure is promoted by their strong uniaxial magnetic anisotropy. Consequently, magnetization reversal proceeds by nucleation or depinning of a single DW that propagates long distances under the action of a homogeneous magnetic field. Its velocity reaches values as high as $\mathrm{km} / \mathrm{s}$, and its dynamics and intrinsic damping mechanisms have been thoroughly investigated recently [18-20]. Here, we report on the effect of local magnetic fields on that moving wall.

This wire is a nearly ideal system that allows us to design novel experiments to study essential properties of single DWs of relevance in advanced spintronics and 3D logic devices. Present results can be easily extended and applied to nanomagnetic systems because general principles are used.

A simple, Sixtus \& Tonks-like, experiment was designed to demonstrate the controlled motion of a single DW as is schematically shown in Fig. 1(a). The setup consists of $11 \mathrm{~cm}$ long solenoid which creates a uniform driven magnetic field, $H_{\mathrm{dr}}$, (leaving a stable remanence domain structure characterized by a long axial domain and closure structures at the ends) and a short (4 $\mathrm{mm}$ long) exciting coil generating a local longitudinal field, $H_{L}$, favoring or opposing $H_{\mathrm{dr}}$, thus affecting the motion of the propagating wall. This coil was placed exactly at the middle of the main solenoid and equidistant from two pickup coils $(3 \mathrm{~mm}$ long) symmetrically placed and separated $87 \mathrm{~mm}$. Both, the drive and the local-field coils were in phase fed by $40 \mathrm{~Hz}$ frequency ac square electrical current. That frequency is low enough so that the rising of the field signal reaches its stationary value and a constant field is assumed to act on the propagating walls. Note in Fig. 1(a) that the wire is asymmetrically placed, which generates a local magnetic inhomogeneity at its left-hand end. Consequently, under a switching reverse field, a domain wall depins from the left end and propagates to right. The rightward motion of the DW is confirmed by the sequence of sensed pulses in the pickup coils. The methodology for DW velocity determination can be found elsewhere 

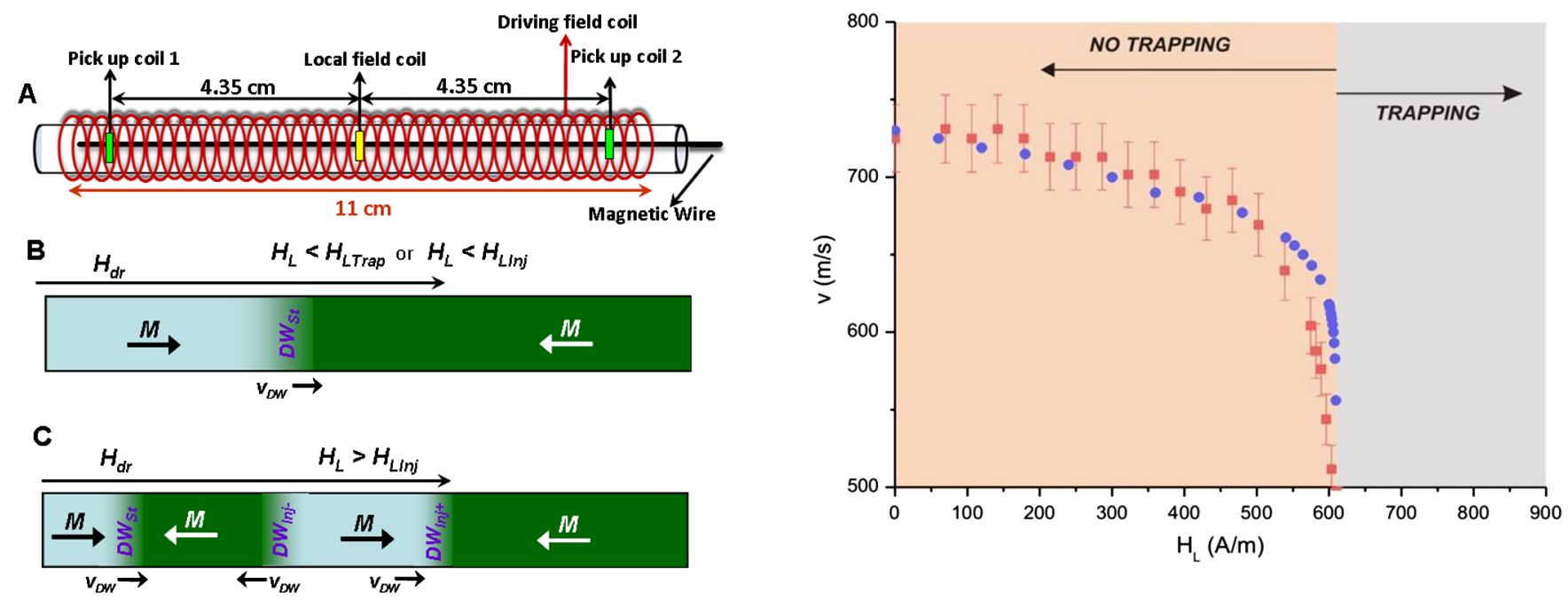

FIG. 1 (color online). General view of the coils system and the microwire (a). Schematic view of the domain structure during DWs propagation under drive field, $H_{\mathrm{dr}}$ : standard wall, $\mathrm{DW}_{\mathrm{St}}$, propagating under additional reduced local field, $H_{L}\left(H_{L}=0\right.$, $H_{L}<H_{\mathrm{LTrap}}$, in antiparallel configuration, or $H_{L}<H_{\mathrm{LInj}}$, in parallel configuration) (b). Standard, $\mathrm{DW}_{\mathrm{St}}$, and injected, $\mathrm{DW}_{\text {Inj- }}$ and $\mathrm{DW}_{\text {Inj+ }}$, walls moving under strong parallel local field $\left(H_{L}>H_{\text {LInj }}\right)$. Arrows denote the magnetization, $M$, orientation inside domains and the DWs propagation velocity, $v_{\text {DW }}(\mathrm{c})$.

[17,19,21-24]. Under a homogeneous drive field, a standard DW depins from the left end of the wire and its speed is evaluated from the time interval between induced signals in the sensing coils. A fast DW propagation is observed, with a linear dependence of its velocity on the drive field ranging between 750 and $840 \mathrm{~m} / \mathrm{s}$ when the drive field increases from 160 to $300 \mathrm{~A} / \mathrm{m}$. This expected linear behavior is represented by the motion equation: $v=$ $\mu\left(H_{\mathrm{dr}}-H_{0}\right)$, where $v$ is DW velocity, $\mu=2 \mu_{0} M_{s} / \beta$ its mobility ( $\beta$, the effective damping parameter), and $H_{0}$ denotes a minimum applied field required for the DW propagation.

In the case of the antiparallel field configuration $\left(H_{\mathrm{dr}}-\right.$ $H_{L}$ ), the standard domain wall, $\mathrm{DW}_{\mathrm{St}}$, generated at the left end moves to the right, reaches the first sensing coil, and then approaches the local coil. $H_{L}$ brakes its motion as is observed in Fig. 2. Moreover, for a given local-field amplitude, $H_{L}=H_{\text {LTrap }}$, the wall bounces and is trapped somewhere before reaching the local-field coil, just where the sum of both fields, $H_{\mathrm{dr}}+H_{L}$, is zero. In our particular experiment shown in Fig. 2, under constant $H_{\mathrm{dr}}=$ $170 \mathrm{~A} / \mathrm{m}$, the equilibrium position of the $\mathrm{DW}_{\mathrm{St}}$ is calculated to be at $4.27 \mathrm{~mm}$ from the local coil. This bounce and stop of the $\mathrm{DW}_{\mathrm{St}}$ is directly evidenced by the fact that a peak is only recorded by the left sensing coil while no DW reaches the pickup coil to the right [see Fig. 3(a)].

The case of the parallel field configuration $\left(H_{\mathrm{dr}}+H_{L}\right)$ gives rise also to an interesting outcome. Figure 4(a) shows the DW velocity as a function of $H_{\mathrm{dr}}$ for selected values of

FIG. 2 (color online). The standard DW velocity, $v$, is tuned in with a local field, $H_{L}$, antiparallel to the homogeneous drive field $\left(H_{\mathrm{dr}}=170 \mathrm{~A} / \mathrm{m}\right)$ originating the wall motion [see Fig. 1(b)]. Notice that for a given local field, $H_{L}=H_{\text {LTrap }} \approx 610 \mathrm{~A} / \mathrm{m}$, the DW gets trapped. Experimental $(\square)$ and calculated $(\bigcirc)$ data of $\mathrm{DW}_{\mathrm{St}}$ speed are included.

$H_{L}$. Note that for $H_{L}=0$, the DW propagates with linearly-increasing velocity proportional to $H_{\mathrm{dr}}$. Also, notice that the DW speed is not affected when increasing $H_{L}$ roughly up to about $H_{L} \leq 1 \mathrm{kA} / \mathrm{m}$, as can be observed in Fig. 4(b). Nevertheless, for a critical value, $H_{L} \approx 1 \mathrm{kA} / \mathrm{m}$, the induced peaks nearly simultaneously reach both sensing coils which are symmetrically placed from the localfield coil [see Fig. 3(b)]. In order to understand such peaks overlapping we should admit that $H_{L}$, added to $H_{\mathrm{dr}}$, is high enough to nucleate a local reverse domain. The nucleation field is thus estimated to be around $1200 \mathrm{~A} / \mathrm{m}$. It implies that two walls, namely, tail-to-tail and head-to-head DWs, $\mathrm{DW}_{\text {Inj- }}$ and $\mathrm{DW}_{\text {Inj+ }}$, respectively, in Fig. 1(c), are injected at $H_{L}=H_{\text {LInj }}$ and almost immediately propagate along opposite orientations reaching the pickup coils nearly simultaneously. Considering the distance from the local-field coil to the sensing coils and the time interval between switching on $H_{L}$ and reaching the sensing coils, we can estimate a lower limit for the velocity of these injected walls as it is given in Figs. 4(a) and 4(b). As observed in Fig. 4(b), the injected DW apparently propagates at nearly $1 / 2$ speed compared with the standard DW. We should emphasize, nevertheless, that the injected DW velocity given value should actually be understood as a lower limit owing to the uncertainty in the determination of the instant when the walls properly start propagating and that we are computing this wall dynamics transient process with the same speed as the final stationary regime.

We should notice that during some time interval, three DWs (the standard, $\mathrm{DW}_{\mathrm{St}}$, and the injected $\mathrm{DW}_{\text {Inj- }}$ and $\mathrm{DW}_{\text {Inj }}+$ walls in Fig. 1(c)] are simultaneously propagating although the left-hand sensing coil will only detect the signal from the wall arriving first, either the standard 

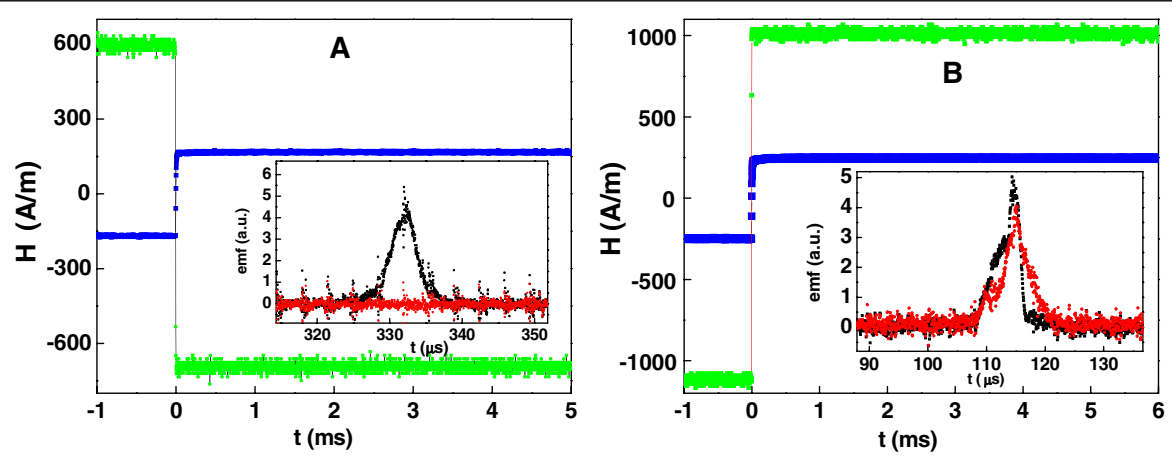

FIG. 3 (color online). Drive, $H_{\mathrm{dr}}$ (dark grey in printed version, blue online), and (high-amplitude) Local, $H_{L}$ (light grey in printed version, green online), fields, and emf responses recorded in the left (grey in printed version, red online) and right (black) sensing coils: antiparallel (a) and parallel (b) field configurations.

one, $\mathrm{DW}_{\mathrm{St}}$, coming from the left end or the tail-to-tail wall, $\mathrm{DW}_{\text {Inj- }}$, coming from the middle of the wire.

Complementary calculations on the influence of the local field on the DW dynamics confirm that an antiparallel local field results in the braking and finally trapping of the standard wall. The equation of motion of a wall can be written as:

$$
m \frac{d^{2} x}{d t^{2}}+\beta \frac{d x}{d t}+k x=2 \mu_{0} M_{s} H S
$$

where $m$ is the inertial mass of the wall, $\beta$ the damping coefficient, $k$ the restoring force constant, $\mu_{0}$ the permeability of vacuum, $M_{s}$ the saturation magnetization of the wire, $H$ the total applied field, and $S$ the section of the DW.

In our experiment the applied magnetic field, $H_{\mathrm{ap}}$, contains two terms:

$$
H_{\mathrm{ap}}=H_{\mathrm{dr}}+H_{L} \text {, }
$$

the drive field, $H_{\mathrm{dr}}$, is spatially constant during the experiment and the local field, $H_{L}$, can be expressed as:

$$
H_{L}=\frac{N I}{2 l}\left[\frac{x+l / 2}{\sqrt{(x+l / 2)^{2}+a^{2}}}-\frac{x-l / 2}{\sqrt{(x-l / 2)^{2}+a^{2}}}\right],
$$

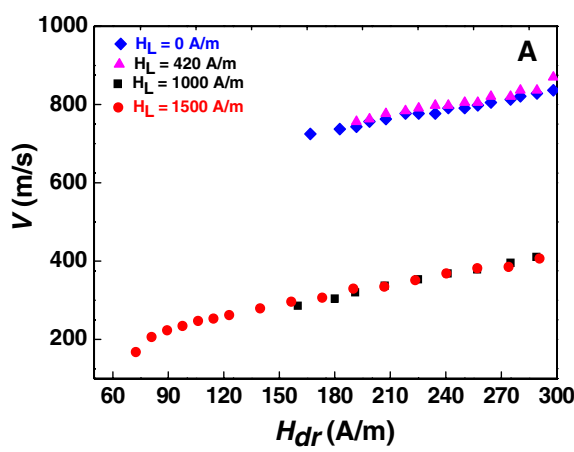

where $I$ is the current amplitude through the local coil, characterized by $N$ turns, length $l$, and radius $a$. The origin of the $x$ coordinate is taken at the center of the coil. Equation (1) can be then expressed as:

$$
m v \frac{d v}{d x}+\beta v+k x=2 \mu_{0} M_{s} S\left(H_{\mathrm{dr}}+H_{L}(x)\right)
$$

The restoring force, $k x$, does not have a local influence but it is usually assumed to be an average of the constant resistance to the wall motion due to defects. However, for this kind of materials, this term is negative acting as an additional force or apparent magnetic field [18]. In any case, from the mathematical point of view we can consider:

$$
m v \frac{d v}{d x}+\beta v=2 \mu_{0} M_{s} S\left[H_{d}+H_{L}(x)\right]+F_{\text {res }}
$$

This differential equation has been solved by means of MATLAB $6.5 \AA$, using the code 45 algorithm for the RungeKutta method. From Fig. 4 we obtain that $\beta=$ $3.69 \times 10^{-10} \mathrm{~kg} \mathrm{~s}^{-1}$. The mass of the DW (which cross section is taken as that of the wire) is evaluated to be $m=$ $3.257 \times 10^{-15} \mathrm{~kg}$ after considering the values of the applied fields to stop the domain wall $\left(H_{\mathrm{dr}}=610 \mathrm{~A} / \mathrm{m}\right.$, and

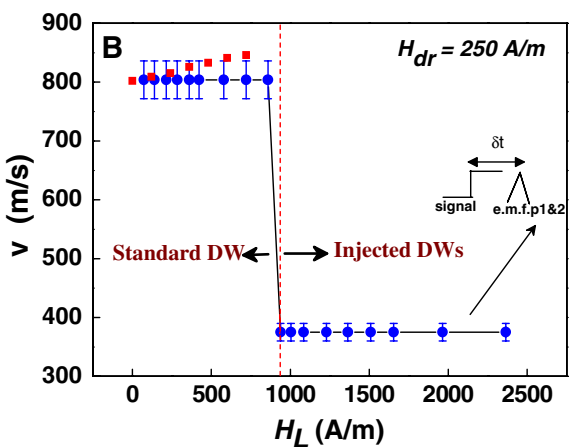

FIG. 4 (color online). Parallel field configuration. a) Wall velocity as a function of $H_{\mathrm{dr}}$ for selected values of $H_{L}$. b) Wall velocity as a function of $H_{L}$ under constant drive field $\left(H_{\mathrm{dr}}=250 \mathrm{~A} / \mathrm{m}\right)$. Above a critical local field, $H_{L}>H_{\text {LInj }}$, the velocity is estimated from the time interval between the switching on of the local field and the reaching of the pickup coil. 

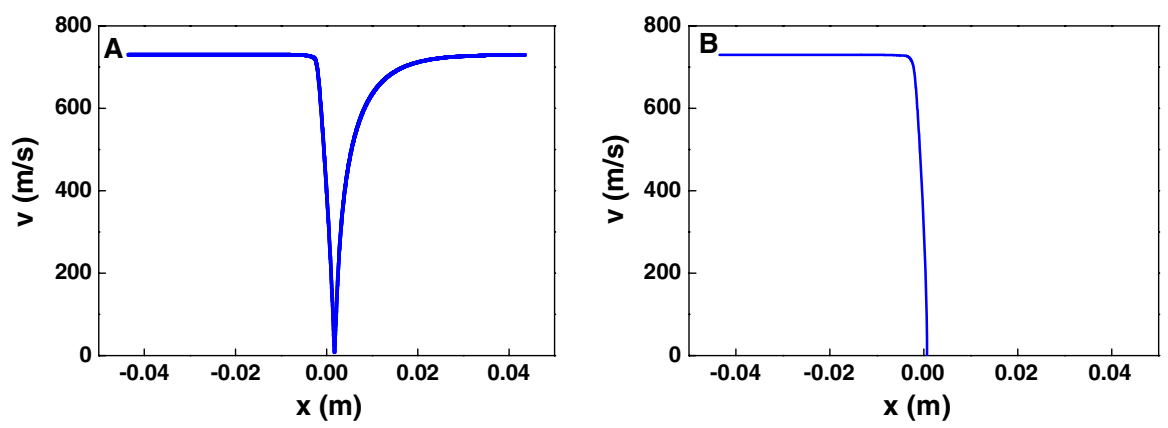

FIG. 5 (color online). Calculated velocity of the standard DW as a function of the position between pickup coils for antiparallel to $H_{\mathrm{dr}}$ : a) $H_{L}$ is slightly below the critical field to trap the DW $\left(H_{L} \leq H_{\mathrm{LTrap}}\right)$; b) $H_{L}$ is above that critical field $\left(H_{L}>H_{\mathrm{LTrap}}\right)$.

$H_{\text {LTrap }}=170 \mathrm{~A} / \mathrm{m}$, see Fig. 2) and the initial velocity $(730 \mathrm{~m} / \mathrm{s})$.

Figure 5. A shows the calculated velocity of the domain wall as it moves from pickup coil 1 to pickup coil 2 under a local field, $H_{L}$, antiparallel to the drive field, $H_{\mathrm{dr}}$, and slightly smaller than the critical value to trap the domain wall. In the first region, the domain wall moves with a constant velocity as the influence of the local magnetic field is constrained to a small region around the local coil. The decreasing of the wall velocity is then very abrupt, being nearly stopped in a position above zero from the center of the system. However, the local magnetic field is not strong enough to completely stop the domain wall and it finally reaches the equilibrium velocity close to the pickup coil 2. The velocity of the DW is asymmetrical due to the influence of the damping and the inertial mass.

Above the critical value of the local magnetic field, the differential Eq. (5) only has a solution for a certain range of positions, as is shown in Fig. 5(b). The DW bounces and, owing to its inertia, gets back towards the first pickup coil until it is stopped at the zero field position, which for the system here described, corresponds to $-4.27 \times 10^{-3} \mathrm{~m}$ (measured from the center of the system).

The calculated influence of the local field on the DW speed for the antiparallel field configuration (driving field $170 \mathrm{~A} / \mathrm{m}$ ) fits very well with the experimental data (see Fig. 2). In the case of parallel field configuration (equilibrium velocity of $804 \mathrm{~m} / \mathrm{s}$ and driving field of $250 \mathrm{~A} / \mathrm{m}$ ), a slight increase of the velocity of the DW is obtained which is not observed in the experimental results as it remains nearly within the experimental error bar [see Fig. 4(b)].

The calculated velocity values as $H_{L}$ increases are included in Fig. 2. The critical field to trap the standard domain wall, $\mathrm{DW}_{\mathrm{St}}$, can be reduced tailoring both the damping coefficient of the DW as well as its inertial mass, related to the volume of the DW, which extends these results to smaller geometries.

Some implications can be derived from the present experiment particularly on the braking and trapping of DWs as well as of the nucleation of local domains with the corresponding injection of pairs of tail-to-tail and head-to-head DWs. Injection of DWs can be achieved by profiting local moment distribution around local defects that transform into local reverse domains under the action of suitable magnetic fields. This is also the case of the magnetic moment inhomogeneities generated at the ends of our wire to diminish the stray fields energy. Here, in addition, we show that a local magnetic field can be properly employed to trap or inject DWs conveniently.

The control of trapping and eventual subsequent motion activation of DWs is in the very essence of advanced race track memory devices. The related experience offers novel possibilities to be open, for example, by combining the opposite effect of sets of such local coil fields distributed along the wire. One set coil generating local fields, $H_{L 1}$ (parallel configuration) would inject pairs of walls moving in opposite directions while the other one, generating a local field, $H_{L 2}<H_{L 1}$ (antiparallel configuration and smaller amplitude) would trap walls moving in one of the given directions. In this multi-injection of head-to-head and tail-to-tail DWs, the field pulses for nucleating, trapping, and driven fields should be reversed in a convenient sequence. Ideal wires are needed, with constant roughness and magnetoelastic stresses distribution at the surface.

The results presented here are for a cylindrical magnetic wire a few micrometers in diameter. Nevertheless, the consequences are straightforward and can be extended for wires with reduced diameters and different cross sections. Lithography methods can be properly used for preparing tiny exciting coils to inject and trap DWs which, together with suitable sensing heads, could be used for storage of magnetic information.

This work has been performed under support of the Spanish Ministry of Science and Innovation, Project No. MAT2010-20798-C05-01. G. A. Basheed acknowledges the postdoctoral grant from the Spanish MICINN.

*correspondence to: mvazquez@icmm.csic.es

[1] S. D. Bader and S. S. P. Parkin, Annu. Rev. Condens. Matter Phys. 1, 71 (2010).

[2] T. Ono, H. Miyajima, K. Mibu, N. Hosoito, and T. Shinjo, Science 284, 468 (1999). 
[3] C. K. Lim, T. Devolder, C. Chappert, J. Grollier, V. Cros, A. Vaurès, A. Fert, and G. Faini, Appl. Phys. Lett. 84, 2820 (2004).

[4] S. S. P. Parkin, M. Hayashi, and L. Thomas, Science 320, 190 (2008).

[5] D. A. Allwood, G. Xiong, C. C. Faulkner, D. Atkinson, D. Petit, and R. P. Cowburn, Science 309, 1688 (2005).

[6] M. Hayashi, L. Thomas, C. Rettner, R. Moriya, X. Jiang, and S.S.P. Parkin, Phys. Rev. Lett. 97, 207205 (2006).

[7] M. Kläui, C. A. F. Vaz, J. A. C. Bland, W. Wernsdorfer, G. Faini, E. Cambril, L. J. Heyderman, F. Nolting, and U. Rüdiger, Phys. Rev. Lett. 94, 106601 (2005).

[8] L. K. Bogart, D. Atkinson, K. O'Shea, D. McGrouther, and S. McVitie, Phys. Rev. B 79, 054414 (2009).

[9] D. Djuhana, H.-G. Piao, S.-H. Lee, D.-H. Kim, S.-M. Ahn, and S.-B. Choe, Appl. Phys. Lett. 97, 022511 (2010).

[10] L. O’Brien, D. Petit, E. R. Lewis, R. P. Cowburn, D. E. Read, J. Sampaio, H. T. Zeng, and A.-V. Jausovec, Phys. Rev. Lett. 106, 087204 (2011).

[11] M.-Y. Im, L. Bocklage, P. Fischer, and G. Meier, Phys. Rev. Lett. 102, 147204 (2009).

[12] M. T. Bryan, T. Schrefl, D. Atkinson, and D. A. Allwood, J. Appl. Phys. 103, 073906 (2008).

[13] K. Richter, R. Varga, G. A. Badini-Confalonieri, and M. Vázquez, Appl. Phys. Lett. 96, 182507 (2010).
[14] S. Allende, D. Altbir, E. Salcedo, M. Bahiana, and J. P. Sinnecker, J. Appl. Phys. 104, 013907 (2008).

[15] A. Kunz and S.C. Reiff, Appl. Phys. Lett. 93, 082503 (2008).

[16] M. Vazquez, H. Chiriac, A. Zhukov, L. Panina, and T. Uchiyama, Phys. Status Solidi A 208, 493 (2011).

[17] M. Vázquez, Handbook of Magnetism and Advanced Magnetic Materials, edited by $\mathrm{H}$. Kronmüller and S.S.K. Parkin (Wiley, Chichester, West Sussex, England, 2007), Vol. 4, p. 2193.

[18] R. Varga, K. L. Garcia, M. Vázquez, and P. Vojtanik, Phys. Rev. Lett. 94, 017201 (2005).

[19] A. Zhukov and V. Zhukova, Magnetic Properties and Applications of Ferromagnetic Microwires with Amorphous and Nanocrystalline Structure (Nova Science Publishers, Inc., Hauppauge, NY, 2009), Vol. 162, p. 11788.

[20] T. A. Ovari, S. Corodeanu, and H. Chiriac, J. Appl. Phys. 109, 07D502 (2011).

[21] H. Chiriac, S. Corodeanu, M. Lostun, G. Ababei, and T. A. Ovari, J. Appl. Phys. 107, 09A301 (2010).

[22] G. Infante, R. Varga, G. A. Badini-Confalonieri, M. Vázquez, Appl. Phys. Lett. 95, 012503 (2009).

[23] P. A. Ekstrom and A. Zhukov, J. Phys. D 43, 205001 (2010).

[24] V. Zhukova, J. M. Blanco, M. Ipatov, and A. Zhukov, J. Appl. Phys. 106, 113914 (2009). 